\title{
Primordial black holes formation from particle production during inflation
}

To cite this article: Encieh Erfani JCAP04(2016)020

View the article online for updates and enhancements.

\section{Related content}

- Gauge-preheating and the end of axion
$\frac{\text { inflation }}{\text { Peter Adshead, John T. Giblin Jr., Timothy }}$
R. Scully et al.
- Primordial black holes and associated
$\frac{\text { gravitational waves in axion monodromy }}{\text { inflation }}$
Shu-Lin Cheng, Wolung Lee and Kin-
Wang Ng
- Fourth level MSSM inflation from new flat
$\frac{\text { directions }}{\text { Sayantan Choudhury and Supratik Pal }}$

\section{Recent citations}

- Nonlinear perturbations from axion-gauge
$\frac{\text { fields dynamics during inflation }}{\text { Alexandros Papageorgiou et al }}$
- Nonlinear perturbations from the coupling
$\frac{\text { of the inflaton to a non-Abelian gauge }}{\text { field, with a focus on Chromo-Natural }}$
$\frac{\text { Inflation }}{\text { Alexandros Papageorgiou et al }}$
- Primordial black holes and associated
$\frac{\text { gravitational waves in axion monodromy }}{\text { inflation }}$
Shu-Lin Cheng et al

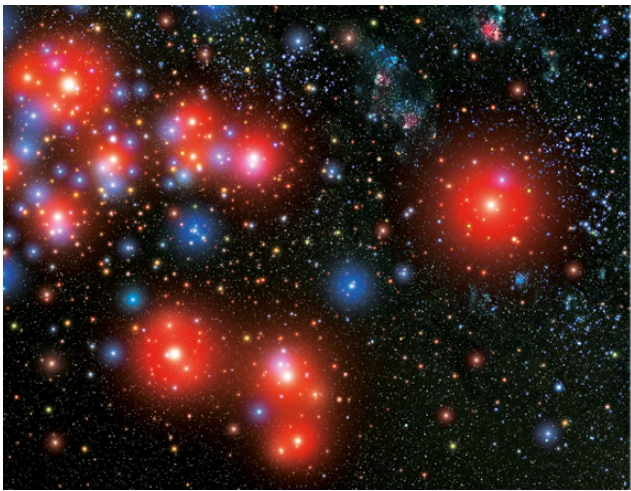

\section{A A IOP Astronomy ebooks}

Part of your publishing universe and your first choice for astronomy, astrophysics, solar physics and planetary science ebooks. iopscience.org/books/aas 


\title{
Primordial black holes formation from particle production during inflation
}

\section{Encieh Erfani}

Department of Physics, Institute for Advanced Studies in Basic Sciences (IASBS), No. 444, Prof. Yousef Sobouti Blvd., Zanjan, Iran

ICTP South American Institute for Fundamental Research, IFT-UNESP, Rua Dr. Bento Teobaldo Ferraz 271, Sao Paulo, Brazil

E-mail: erfani@iasbs.ac.ir

Received January 19, 2016

Revised March 14, 2016

Accepted March 25, 2016

Published April 11, 2016

\begin{abstract}
We study the possibility that particle production during inflation can source the required power spectrum for dark matter $(\mathrm{DM})$ primordial black holes $(\mathrm{PBH})$ formation. We consider the scalar and the gauge quanta production in inflation models, where in the latter case, we focus in two sectors: inflaton coupled $i$ ) directly and $i$ i) gravitationally to a $U(1)$ gauge field. We do not assume any specific potential for the inflaton field. Hence, in the gauge production case, in a model independent way we show that the non-production of DM PBHs puts stronger upper bound on the particle production parameter. Our analysis show that this bound is more stringent than the bounds from the bispectrum and the tensor-toscalar ratio derived by gauge production in these models. In the scenario where the inflaton field coupled to a scalar field, we put an upper bound on the amplitude of the generated scalar power spectrum by non-production of PBHs. As a by-product we also show that the required scalar power spectrum for $\mathrm{PBHs}$ formation is lower when the density perturbations are non-Gaussian in comparison to the Gaussian density perturbations.
\end{abstract}

Keywords: inflation, primordial black holes

ArXiv ePrint: 1511.08470 


\section{Contents}

1 Introduction $\quad 1$

2 Constraints from dark matter primordial black holes $\quad 3$

3 Particle production during inflation models 5

3.1 Gauge production $\quad 7$

3.1.1 Model I: inflaton coupled directly to the gauge field 8

3.1.2 Model II: inflaton coupled gravitationally to the gauge field 10

$\begin{array}{lll}3.2 & \text { Scalar production } & 11\end{array}$

4 Summary and conclusions

\section{Introduction}

Inflation is currently the standard paradigm for solving the cosmological puzzles of the standard big bang cosmology, such as homogeneity, isotropy and flatness of the universe. In addition, the quantum fluctuation of the inflaton field explain the generation of all (classical) inhomogeneities that can be seen in our universe, from the Cosmic Microwave Background (CMB) anisotropies to the Large Scale Structure (LSS) [1]. In the simplest models, inflaton field slowly rolls down its potential, however, generically the inflaton should be expected to couple to some additional degrees of freedom and a variety of different models have been proposed. ${ }^{1}$

Recently, inflation models where production of some non-inflaton particles happens during inflation via parametric resonance has received a lot of attention ${ }^{2}[4-6,8-11]$. In this class of models, the inflaton field couples to another field directly or gravitationally and the coupled field can be massless or massive fermion [4], scalar [5-7] or gauge field [12-14]. The production of light species occurs during inflation at the expenses of the kinetic energy of the inflaton and slows down its motion; i.e. resonant extraction of inflaton field energy decreases $\dot{\phi}$, leading to an increase in the scalar power spectrum, $\mathcal{P}_{\zeta} \propto H^{4} / \dot{\phi}^{2}$. It was shown that the production of particles during inflation provides a qualitatively new mechanism for generating cosmological perturbations $[4,8,9]$. The nature of these fluctuations is usually non-scale-invariant and non-Gaussian (NG) [13-16].

A particular feature is that the power spectrum of these fluctuations can be very blue; this means that the amplitude of density fluctuations can be much higher at the small length scales relevant for Primordial Black Holes (PBHs) formation [17, 18]. To be consistent with cosmological observations such as the CMB and the LSS, these fluctuations should not dominate the primordial density perturbation at large scales. However, depending on individual models, their significant contribution to the non-Gaussianity is still possible. In this paper, we will show that these fluctuations with a blue spectrum in the very small scales, can seed the formation of Dark Matter (DM) PBHs, therefore their non-production

\footnotetext{
${ }^{1}$ In general, the coupling of the inflaton to additional degrees of freedom is necessary for reheating scenario after inflation $[2,3]$.

${ }^{2}$ The phenomenon of resonant production of particles has received much attention in the past for successful (pre-)reheating after inflation.
} 
can constraint the particle production parameter which is a function of the parameter(s) of the inflation model.

Here, we will study the coupling of the inflaton to a massless scalar and a gauge field. In [6], a simple model where scalar $\chi$ particles are produced through a coupling $g^{2} \phi^{2} \chi^{2}$ to the inflaton, $\phi$ is considered. In this model, the $\chi$ field whose mass depends on the inflaton becomes effectively massless as the inflaton rolls down its potential, therefore it becomes energetically cheap to produce its quanta. In this case, instantaneous bursts of particle production leads to localized (bump-like) feature in the cosmological perturbations. We will show that the non-production of PBHs with mass $\sim 10^{15} \mathrm{~g}-$ as candidate for DM - can put an upper bound on the amplitude of these features. ${ }^{3}$

Another possibility is that particle production occurs continuously during inflation. This can happen when the inflaton, $\phi$ couples to a derivative of some field such as a gauge field $[8,12,15]$. This is quite natural in the context of axion inflation [20] via the coupling of the pseudoscalar field to gauge fields, $\alpha \phi F \tilde{F}$. This interaction leads to a continuous tachyonic production of gauge field fluctuations during inflation if the coupling, $\alpha$ is large enough. It has been shown $[13,15]$ that, these gauge fields through their inverse decay into inflaton perturbations - i.e. $\delta A+\delta A \rightarrow \delta \phi-$ can affect all scalar primordial $N$-point functions. The effect on the power spectrum is a blue tilted contribution, which grows on smaller scales. Since the inverse decay produces NG inflaton perturbations, the bispectrum is non-vanishing and peaks on equilateral configurations. In addition, gauge fields production can also leads to observable effects in the tensor power spectrum [14, 19, 21]. The effect is negligible at the CMB scales but it could be detected by gravitational wave (GW) interferometers such as advanced LIGO/Virgo $[22,23] .^{4}$

Since the inverse decay of gauge fields into scalar perturbations leads to a blue tilt in the primordial scalar power spectrum it is natural to ask what the constraint from the PBHs formation is. As we will see in section 3, at very small scales, the produced gauge quanta largely increases the curvature power spectrum which can reach $\mathcal{P}_{\zeta} \sim \mathcal{O}\left(10^{-4}\right)$ required for DM PBHs formation. Therefore, the non-production of such PBHs can put an upper bound on the scalar and the tensor power spectra and the bispectrum.

The rest of the paper is organized as follows: in section 2, we present a brief review of the Press-Schechter formalism [25] describing PBH formation by considering the new threshold derived in [26] for their formation. We will focus on non-evaporating PBHs as candidate for DM and we will discuss the choice of a Gaussian and a non-Gaussian probability distribution function (PDF) for the perturbations as seed for their formation. And we will derive the required power spectrum for the production of such PBHs in both cases. We will show that when the fluctuations are NG, the required spectral index (hence, the power spectrum) in the scale of DM PBHs is lower than the case when the perturbations are Gaussian. Therefore, the probability of DM PBHs formation is higher in the NG case.

In section 3, we will review the particle production during inflation in different inflation models and we will estimate the scalar and the tensor power spectra and the bispectrum, and we will explore the possibility of DM PBHs formation in these models. In section 3.1, we will review the main formulas and results of the axion inflation model in which the inflationary expansion is accompanied by the gauge quanta production. We will consider both cases when

\footnotetext{
${ }^{3}$ In this case, the particle production can also leads to observable effects in the tensor power spectrum [19]. However, in this paper we are not interested in this effect.

${ }^{4}$ In [24], they claimed that particle production during inflation can result in a GW detectable in the CMB scale.
} 
the inflaton couples directly and gravitationally to the gauge field. Our consideration differs from the consideration carried out in the recent works [27-29] in two respects. Firstly, we will not assume any specific potential for the inflaton (or coupled) field. Secondly, we are only interested in DM PBHs; i.e. the ones with mass $\gtrsim 10^{15} \mathrm{~g}$. We will show that in these cases the non-production of DM PBHs at the end of inflation, puts stronger upper bound on the particle production parameter, $\xi$. And this bound is more stringent than the bounds from the bispectrum and the tensor-to-scalar ratio derived by gauge production in these models.

We will also review a mechanism analogous to that of section 3.1, where the gauge field is replaced by a massless scalar field in section 3.2. In a model independent way, we will put an upper bound on the amplitude of the (bump-like) feature of power spectrum - generated by scalar production during inflation - via the non-production of DM PBHs in the CMB observational range.

Finally, in section 4 we present our conclusions.

Through this paper, we use the units $c=\hbar=1$.

\section{Constraints from dark matter primordial black holes}

Primordial black holes may have formed from primordial fluctuations in the early universe [30, 31]. Actually, there is no conclusive evidence for their existence and upper bounds on their abundance on various mass scales have been obtained by various kinds of observations (for recent results, see [32]). These upper bounds can be translated into upper bounds on the power spectrum of the scalar perturbation on comoving scales much smaller than those relevant to the CMB; i.e. they can be used to exclude inflationary models which predict too many PBHs $[17,18] .{ }^{5}$ On the other hand, according to the Hawking radiation [34] the PBHs with mass larger than $10^{15} \mathrm{~g}$ which survive till present time could be candidate for DM.

The traditional treatment of PBH formation is based on the Press-Schechter formalism [25] and in [17], this mechanism has been studied in detail for the formation of DM PBHs. Here, we briefly review this formalism by considering the new derived threshold required for their formation. We will derive the required power spectrum for the production of DM PBHs in two cases: where the perturbations follow a $i$ ) Gaussian and a ii) non-Gaussian distribution.

PBHs will form if at horizon re-entry, the amplitude of density perturbation passes a threshold value, $\zeta_{\text {th }} \equiv(\delta \rho / \rho)_{\text {th }}$. The correct value of the threshold is quite uncertain. It was first provided by Carr [35], giving $\zeta_{\text {th }} \simeq w$, where $w$ is the parameter of the equation of state, $p=w \rho$. Recently, in [26], a new analytic formula for the threshold required for PBHs formation in the universe dominated by a perfect fluid is derived. According to this formula, the amplitude of the density perturbations in the comoving hypersurfaces is given by $\zeta_{\text {th }}=$ $\frac{3(1+w)}{5+3 w} \delta_{\mathrm{H}}^{\mathrm{UH}}$, where $\delta_{\mathrm{H}}^{\mathrm{UH}}=\sin ^{2}(\pi \sqrt{w} /(1+3 w))$ is the amplitude of the density perturbations at the horizon crossing time in the uniform Hubble slice. Therefore, $\zeta_{\text {th }} \simeq 0.4135$ for a radiation fluid, $w=1 / 3{ }^{6}$

The fraction of the energy density of the universe in which the density fluctuation exceeds the threshold for PBH formation which will hence end up in PBHs with mass $\geq \gamma M,{ }^{7}$

\footnotetext{
${ }^{5}$ Recently, in [33], they claimed that $\mathrm{PBH}$ s can also constrain the amplitude of primordial tensor fluctuations.

${ }^{6}$ Since the produced particles during inflation are massless, we can assume that the results of the PBHs formation in the radiation dominated (RD) era after inflation is applicable in this case, too.

${ }^{7}$ The PBH mass is a fraction $\gamma$ of the horizon mass corresponding to the smoothing scale. A simple analytical calculation suggest $\gamma \simeq w^{3 / 2} \simeq 0.2$ during the radiation era [35].
} 
is given $b^{8}$

$$
f(\geq M)=\gamma \int_{\zeta_{\text {th }}}^{\infty} P(\zeta ; M(R)) \mathrm{d} \zeta
$$

Here $P(\zeta ; M(R))$ is the PDF of the linear density field $\zeta$ smoothed on a scale $R$.

Hawking evaporation and present day gravitational effects constrain this fraction. For PBHs contributing to DM today, they saturate the DM relic density if $f \simeq 5 \times 10^{-19}$ [32].

For a derivation of the PBH constraints we need an expression for the PDF of the smoothed $\zeta$-field. The standard assumption is that it is Gaussian; i.e.

$$
P_{\mathrm{G}}(\zeta ; R)=\frac{1}{\sqrt{2 \pi} \sigma_{\zeta}(R)} \exp \left(-\frac{\zeta^{2}}{2 \sigma_{\zeta}^{2}(R)}\right),
$$

where $\sigma_{\zeta}^{2}(R) \equiv\left\langle\zeta^{2}\right\rangle$ is the mass variance of $\zeta$.

Then

$$
f_{\mathrm{G}}=\frac{1}{2} \operatorname{erfc}\left(\zeta_{\mathrm{th}} / \sqrt{2 \sigma_{\zeta}^{2}(R)}\right)
$$

where $\operatorname{erfc}(x) \equiv 1-\operatorname{erf}(x)$ is the complementary error function.

In some inflation models we will study in the next section, $\zeta$ follows a non-Gaussian distribution. Therefore, the simplest assumption which we can use is that the $\zeta$-field is distributed as a square of some Gaussian field, $g$ [37]

$$
\zeta=g^{2}-\left\langle g^{2}\right\rangle \text {. }
$$

Hence, the PDF of $\zeta$ takes the form

$$
P_{\mathrm{NG}}(\zeta ; R)=\frac{1}{\sqrt{2 \pi\left(\zeta+\sigma_{g}^{2}(R)\right)} \sigma_{g}(R)} \exp \left(-\frac{\zeta+\sigma_{g}^{2}(R)}{2 \sigma_{g}^{2}(R)}\right) .
$$

Note that here, $\sigma_{\zeta}^{2}(R)=2\left\langle g^{2}\right\rangle^{2} \equiv 2\left(\sigma_{g}^{2}(R)\right)^{2}$. Therefore, the fraction of space that can collapse to form black holes in a case $\zeta$ dose not follow a Gaussian distribution is given by

$$
f_{\mathrm{NG}}=\operatorname{erfc}\left(\sqrt{\zeta_{\mathrm{th}}+\sigma_{g}^{2}(R)} / \sqrt{2 \sigma_{g}^{2}(R)}\right) .
$$

Following the procedure in ref. [17], we show the final result in figure 1. Here we have fixed $\zeta_{\text {th }}=0.4135$, and show the results for Gaussian (solid curve) and non-Gaussian (dashed curve) probability distribution function. We found that for the formation of DM PBHs from density perturbations with a power-law spectrum, the spectral index at scale of PBHs formation $\left(k_{\mathrm{PBH}}\right)^{9}$ should be at least $1.418,{ }^{10}$ (1.322) for Gaussian (non-Gaussian) PDF. Therefore, the DM PBHs formation can only happen if the spectral index increases significantly between the scales probed by the CMB and the relevant scales for DM PBHs. With $n_{\mathrm{s}}\left(k_{\mathrm{PBH}}\right) \simeq 1.418(1.322)$, the power spectrum at the scale of DM PBHs formation should be $\mathcal{P}_{\zeta}\left(k_{\mathrm{PBH}}\right) \simeq 2 \times 10^{-2}\left(4 \times 10^{-4}\right)$, which is $\sim 10^{7}\left(10^{5}\right)$ times higher than the value of the power spectrum in the observed CMB scales. Note that for the production of PBHs in the non-Gaussian case, one needs a lower power spectrum in comparison to the Gaussian case.

\footnotetext{
${ }^{8}$ In order to complete this calculation one needs to relate the mass, $M$ to the comoving smoothing scale, $R$ in the RD era (see eq. (2.10) in ref. [17]). In this relation, the pre-factor changes from $5.54 \times 10^{-24}$ to $5.19 \times 10^{-24}$ after the Planck 2015 data [36] due to the change in the matter-radiation equality redshift, $z_{\text {eq }}$.

${ }^{9}$ The relevant scale for the formation of DM PBHs is $k_{\mathrm{PBH}}=1.52 \times 10^{15} \mathrm{Mpc}^{-1}[17]$.

${ }^{10}$ Note that this value is larger than the value found in ref. [17]. This is due to the fact that here the larger threshold value used for the amplitude of the density perturbations at the horizon crossing.
} 


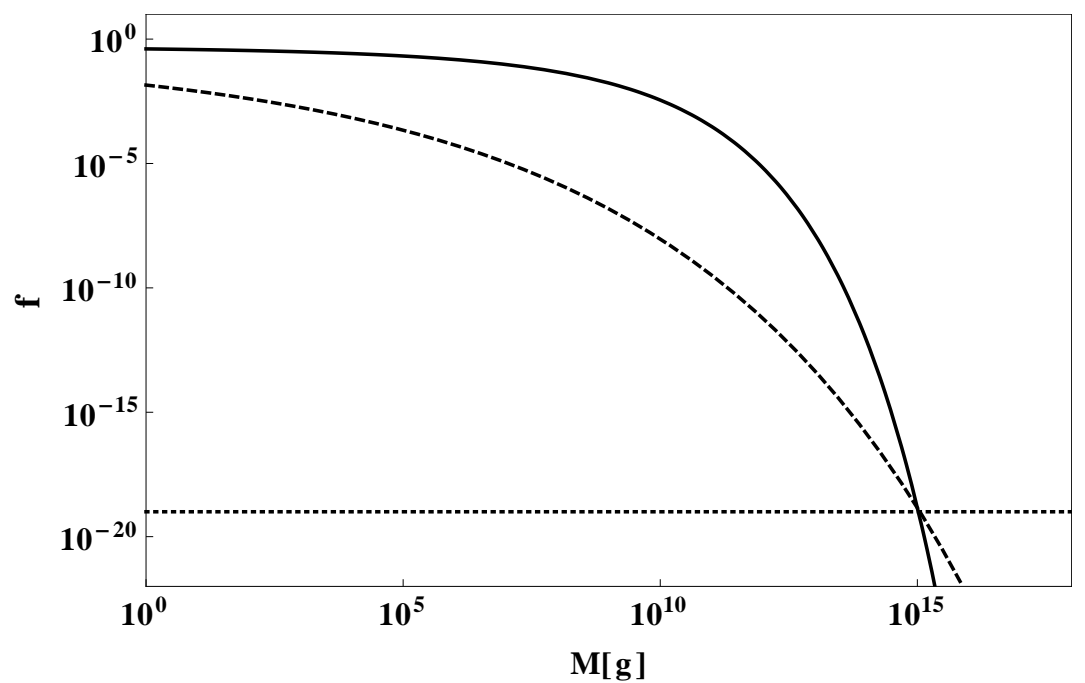

Figure 1. Fraction of the energy density of the universe collapsing into PBHs as a function of the PBH mass for the threshold $\zeta_{\text {th }}=0.4135$. The solid (dashed) curve is for the Gaussian (nonGaussian) probability distribution function. The horizontal dotted line indicates the abundance of DM PBHs, $f \sim 10^{-19}$.

\section{Particle production during inflation models}

In this section, we discuss the fluctuations arised during inflation via the direct or gravitational coupling of the inflaton to another field. We study different inflation models and explore the possibility of DM PBHs formation from these fluctuations.

We consider a model where the slow-rolling inflaton, $\phi$ coupled to a field $\chi \cdot^{11}$ The relevant Lagrangian density is given by

$$
\mathcal{L}(\phi, \chi)=-\frac{1}{2} \partial_{\mu} \phi \partial^{\mu} \phi-V(\phi)-\frac{1}{2} \partial_{\mu} \chi \partial^{\mu} \chi-U(\chi)+\mathcal{L}_{\text {int }}(\phi, \chi),
$$

where $V(\phi)$ is the potential supporting inflation, $U(\chi)$ is the potential of $\chi$ field and the fields interact via $\mathcal{L}_{\text {int }}$ term. ${ }^{12}$

The equations of motion for the inflaton field is given by

$$
\begin{aligned}
H^{2} & =\frac{1}{3 M_{\mathrm{P}}^{2}}\left(\frac{1}{2} \dot{\phi}^{2}+V(\phi)+\rho_{\chi}\right), \\
\ddot{\phi}+3 H \dot{\phi}+V^{\prime}(\phi) & =\frac{\partial \mathcal{L}_{\mathrm{int}}}{\partial \phi}
\end{aligned}
$$

where $\rho_{\chi}$ is the energy density of $\chi$ particles, and the prime and the dot denote differentiating with respect to $\phi$ and time, respectively. ${ }^{13}$ The right hand side of the eq. (3.3), is the backreaction of the interaction to the inflaton mean field which arises due to copious production of $\chi$ quanta during inflation.

\footnotetext{
${ }^{11}$ Through this paper we will denote the inflaton field and the coupled field by $\phi$ and $\chi$, respectively.

${ }^{12} \mathrm{We}$ will assume that only the inflaton potential is dominant during the inflation and we will neglect the potential of the coupled field, $\chi$.

${ }^{13} M_{\mathrm{P}}=1 / \sqrt{8 \pi G} \simeq 2.4 \times 10^{18} \mathrm{GeV}$ is the reduced Planck mass.
} 
In addition, the inflaton fluctuations satisfy

$$
\ddot{\delta \phi}+3 H \dot{\delta \phi}-\frac{\nabla^{2}}{a^{2}} \delta \phi+V^{\prime \prime}(\phi) \delta \phi=\delta\left(\frac{\partial \mathcal{L}_{\text {int }}}{\partial \phi}\right) .
$$

The solution of the above equation without the interaction term gives rise to the primordial density fluctuations and we denote their power spectrum by $\mathcal{P}_{\zeta \text {, vac. }}$ However, the right hand side of eq. (3.4) acts as a source for generating additional fluctuations of $\phi$ and the corresponding power spectrum is denoted by $\mathcal{P}_{\zeta \text {, src. }}$. Hence, the total power spectrum is given by the contributions from both fluctuations as

$$
\mathcal{P}_{\zeta}(k)=\mathcal{P}_{\zeta, \text { vac. }}(k)+\mathcal{P}_{\zeta, \text { src. }}(k) .
$$

From the observational perspective, the key quantities which characterize any model of inflation are the spectrum of scalar and tensor perturbations, $\mathcal{P}_{\zeta}$ and $\mathcal{P}_{\mathrm{t}}$, respectively, along with the bispectrum of scalar perturbations, $B_{\zeta}$ that encodes the leading departures from Gaussian statistics. Therefore, before studying different inflationary models, it is worth defining these cosmological observables and their current constraints.

The power spectrum of the curvature perturbations of inflaton field is given by

$$
\mathcal{P}_{\zeta, \text { vac. }}=\frac{1}{12 \pi^{2} M_{\mathrm{P}}^{6}} \frac{V^{3}(\phi)}{V^{\prime 2}(\phi)}=\frac{1}{24 \pi^{2} M_{\mathrm{P}}^{4}} \frac{V(\phi)}{\epsilon} \simeq \frac{H^{2}}{4 \pi^{2} \dot{\phi}^{2}},
$$

where $\epsilon \equiv \frac{M_{\mathrm{P}}^{2}}{2}\left(\frac{V^{\prime}(\phi)}{V(\phi)}\right)^{2}$ is the first slow-roll parameter.

On the other hand, the simplest assumption for the power spectrum is a scale-free power-law

$$
\mathcal{P}_{\zeta, \text { vac. }}(k)=\mathcal{P}_{\zeta, \text { vac. }}\left(k_{0}\right)\left(\frac{k}{k_{0}}\right)^{n_{\mathrm{s}}(k)-1},
$$

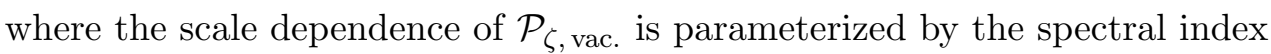

$$
n_{\mathrm{S}}\left(k_{0}\right)-1 \equiv \frac{d \ln \mathcal{P}_{\zeta, \text { vac. }}(k)}{d \ln k} .
$$

During inflation, GW fluctuations are generated by quantum fluctuations of the tensor part of the metric. The power spectrum of these perturbation from vacuum fluctuations during inflation is given by

$$
\mathcal{P}_{\text {t }, \text { vac. }}(k)=\frac{2}{\pi^{2}}\left(\frac{H}{M_{\mathrm{P}}}\right)^{2}\left(\frac{k}{k_{0}}\right)^{n_{\mathrm{t}}},
$$

where $n_{\mathrm{t}}=-2 \epsilon$.

Additional sources of GW may arise due to the coupling of the inflaton to another field. And since the power of the vacuum and the sourced mode are incoherent, they add up [14]

$$
\mathcal{P}_{\mathrm{t}}(k)=\mathcal{P}_{\mathrm{t}, \text { vac. }}(k)+\mathcal{P}_{\mathrm{t}, \text { src. }}(k) .
$$

On the other hand, the tensor contribution to the power spectrum is parameterized by the tensor-to-scalar ratio, $r$

$$
r=\frac{\mathcal{P}_{\mathrm{t}}(k)}{\mathcal{P}_{\zeta}(k)},
$$

and $r=16 \epsilon$ if we consider the primordial scalar and tensor power spectra. 
Non-Gaussian statistics, such as the bispectrum, provide a powerful tool to discriminate between the inflation models and, $f_{\mathrm{NL}}$ characterizes its amplitude

$$
B_{\zeta}\left(k_{1}, k_{2}, k_{3}\right)=f_{\mathrm{NL}} F\left(k_{1}, k_{2}, k_{3}\right),
$$

where the function $F\left(k_{1}, k_{2}, k_{3}\right)$ describes the shape of the bispectrum.

Now let us review the observational bounds on inflation parameters with the Planck "TT, TE, EE+lowP" data [38, 39] at the $68 \%$ confidence level. Note that Planck 2015 full mission data in temperature and polarization are quoted at the pivot scale $k_{0}=0.05 \mathrm{Mpc}^{-1}$.

For the base $\Lambda \mathrm{CDM}$ model, the constraints on the power spectrum, $\mathcal{P}_{\zeta \text {, vac. }}\left(k_{0}\right)$ and the scalar spectral index, $n_{\mathrm{s}}$ are [38]

$$
\begin{aligned}
\ln \left(10^{10} \mathcal{P}_{\zeta, \text { vac. }}\left(k_{0}\right)\right) & =3.094 \pm 0.034 \\
n_{\mathrm{s}} & =0.9645 \pm 0.0049 .
\end{aligned}
$$

The constrain on the tensor-to-scalar ratio inferred from the Planck 2015 data for the $\Lambda \mathrm{CDM}+r$ model is ${ }^{14}[38]$

$$
r_{0.002}<0.10 \quad(95 \% \mathrm{CL}) .
$$

Since in the inflation models we will study in the following section, the inverse decay type NG is present, we only report the observational constraint obtained in Planck 2015 XVII for this type [39]

$$
f_{\mathrm{NL}}=22.7 \pm 25.5
$$

In the rest of the paper, we study the gauge field production in two cases: $i$ ) the inflaton field coupled directly to the gauge field (section 3.1.1) and ii) the inflaton field coupled gravitationally to the gauge field (section 3.1.2). In section 3.2 we consider the scalar particles production via the coupling of the inflaton to a scalar field.

\subsection{Gauge production}

In this section we review the mechanism by which gauge fields are resonantly enhanced when a pseudoscalar field couples to a massless $U(1)$ gauge field. When the coupling is direct, the pseudoscalar field will play the roll of the inflaton [13]. It is also possible that the inflaton field couples gravitationally to a pseudoscalar field which is coupled directly to the gauge field [14].

We can write a generic interaction Lagrangian which is compatible with gauge symmetry as follows [21]

$$
\mathcal{L}_{\text {int }}=-\frac{1}{4} F_{\mu \nu} F^{\mu \nu}-\frac{\alpha}{4 f} \Phi F_{\mu \nu} \tilde{F}^{\mu \nu},
$$

where $\Phi$ could be the inflaton or any other pseudoscalar field and $F_{\mu \nu}=\partial_{\mu} A_{\nu}-\partial_{\nu} A_{\mu}$ is the field strength associated with gauge field, $A_{\mu}{ }^{15}$ and $\tilde{F}_{\mu \nu}=\epsilon_{\mu \nu \alpha \beta} F^{\alpha \beta} / 2$ is its dual. The strength of the interaction is controlled by the dimensionless parameter, $\alpha$ and the decay constant, $f$.

In this type of scenario, gauge fields can be generated during inflation and has a rich and interesting phenomenology both for scalar and tensor primordial fluctuations $[13,16]$.

\footnotetext{
${ }^{14}$ Note that the tensor-to-scalar ratio is reported at $k_{0}=0.002 \mathrm{Mpc}^{-1}$.

${ }^{15}$ For simplicity this gauge field is not the Standard Model one.
} 
Also in this case the inverse decay of gauge field perturbations leads to a large equilateral ${ }^{16}$ contribution to the bispectrum $[13,15]$.

It was shown $[8,13,15]$ that the inverse decay of the gauge field source additional scalar fluctuations to the inflaton field which is given by

$$
\mathcal{P}_{\zeta, \text { src. }}=\gamma_{\mathrm{s}} \epsilon^{2} \mathcal{P}_{\zeta, \text { vac. }}^{2} X^{2}
$$

where $X \equiv \frac{e^{2 \pi \xi}}{\xi^{3}}$ and $\xi \equiv \frac{\alpha}{2 f H} \dot{\Phi}$, is a dimensionless parameter which characterizes the strength of the inverse decay effects. It is clear that when $\xi>0$, the phase space of produced fluctuations experiences a significant exponential enhancement, $e^{\pi \xi} \gg 1$, due to tachyonic instability near horizon crossing.

The produced gauge quanta also source tensor metric perturbations [13] and it was pointed out that these modes are chiral [16]

$$
\mathcal{P}_{\mathrm{t}, \text { src. }}^{ \pm}=\gamma_{\mathrm{t}}^{ \pm} \mathcal{P}_{\mathrm{t}, \text { vac. }}^{2} X^{2},
$$

and, as a consequence of the violation of the parity, the left and right-handed tensor modes have different amplitude of the spectra.

The tensor-to-scalar ratio is as follows

$$
r=\frac{\mathcal{P}_{\mathrm{t}}^{+}+\mathcal{P}_{\mathrm{t}}^{-}}{\mathcal{P}_{\zeta}}
$$

The inverse decay contribution to the primordial cosmological fluctuations is highly NG and is given by

$$
f_{\mathrm{NL}}^{\text {equil. }}=\gamma_{\mathrm{NG}} \epsilon^{3} \mathcal{P}_{\zeta, \text { vac. }} X^{3} .
$$

Note that in eqs. (3.18), (3.19) and (3.21), the coefficients $\gamma_{\mathrm{s}}, \gamma_{\mathrm{t}}$ and $\gamma_{\mathrm{NG}}$ are dimensionless parameters and their precise value needs to be computed in inflation models that we will study in the following sections.

It is clear that in inflation scenarios associated with a pseudoscalar coupling to a $U(1)$ gauge field, the observables (power spectrum, NG and tensor-to-scalar ratio) depend only on the model-dependent quantities $\xi$ and $\epsilon$, which in turn depend on the inflation potential and the dynamics of the coupled field.

In the following we review the inflation models when a pseudoscalar field plays the roll of inflaton or not and we study the possibility of DM PBHs formation in these models. We check whether the bounds from the non-production of such PBHs is stronger than the bounds from NG and tensor perturbations or not. Note that we leave $V(\phi)$ arbitrary, except to suppose that it is sufficiently flat to support $N \gtrsim 60 e$-foldings of inflation.

\subsubsection{Model I: inflaton coupled directly to the gauge field}

We consider a simple theory of a PNGB inflaton interacting directly with a massless $U(1)$ gauge field via the interaction (3.17) [13].

It was shown $[13,15]$, in this model $\gamma_{\mathrm{s}} \simeq 7.5 \times 10^{-5}$ (see eq. (3.17)). Therefore the total scalar power spectrum is given by

$$
\mathcal{P}_{\zeta}=\mathcal{P}_{\zeta, \text { vac. }}\left(1+7.5 \times 10^{-5} \epsilon^{2} \mathcal{P}_{\zeta, \text { vac. }} X^{2}\right) .
$$

\footnotetext{
${ }^{16}$ When a pseudoscalar couples to a vector, non-Gaussianity can feature equilateral configuration because in this model, the source at any moment is dominated by modes with wavelength comparable to the horizon at that moment. This generates mostly correlations between scalar perturbations of comparable size [13].
} 


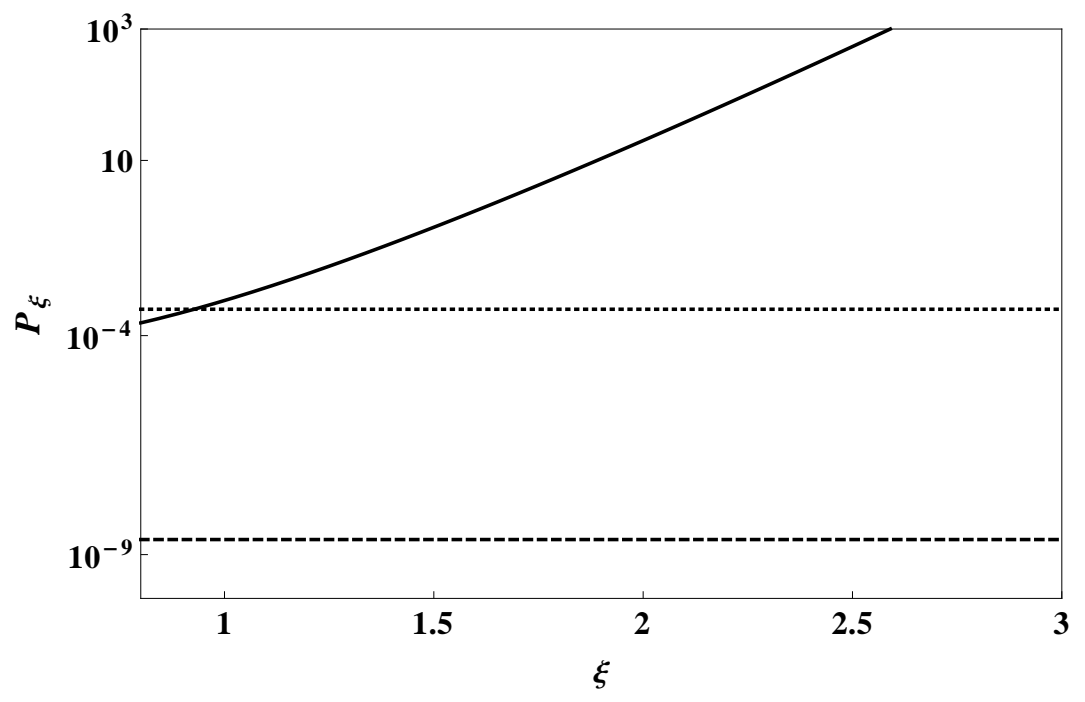

Figure 2. The total curvature power spectrum, $\mathcal{P}_{\zeta}$ as a function of particle production parameter, $\xi$. The DM PBHs bound is the dotted line and the dashed line shows the constraint on the power spectrum from the CMB [38].

For tensor perturbations, it was shown [16] that $\gamma_{t}^{+}=4.3 \times 10^{-7}$ and $\gamma_{t}^{-}=9.2 \times 10^{-10}$. Hence, the overall power spectra of the helicity- \pm components of the GWs generated by this mechanism are as follows

$$
\begin{aligned}
& \mathcal{P}_{\mathrm{t}, \text { src. }}^{+}=4.3 \times 10^{-7} \mathcal{P}_{\mathrm{t}, \text { vac. }}^{2} X^{2}=5.5 \times 10^{-5} \epsilon^{2} \mathcal{P}_{\zeta, \text { vac. }}^{2} X^{2}, \\
& \mathcal{P}_{\mathrm{t}, \text { src. }}^{-}=9.2 \times 10^{-10} \mathcal{P}_{\mathrm{t}, \text { vac. }}^{2} X^{2}=1.2 \times 10^{-7} \epsilon^{2} \mathcal{P}_{\zeta, \text { vac. }}^{2} X^{2},
\end{aligned}
$$

and the tensor-to-scalar ratio is given by

$$
r=16 \epsilon \frac{1+2.2 \times 10^{-7} \mathcal{P}_{\mathrm{t}, \text { vac. }} X^{2}}{1+7.5 \times 10^{-5} \epsilon^{2} \mathcal{P}_{\zeta, \text { vac. }} X^{2}} .
$$

The inverse decay contribution to the primordial cosmological fluctuations lead to equilateral non-Gaussianity in the CMB which is given by [13]

$$
f_{\mathrm{NL}, \zeta}^{\text {equil. }} \approx 4.4 \times 10^{10} \epsilon^{3} \mathcal{P}_{\zeta, \text { vac. }}^{3} X^{3} .
$$

We can plot these results for $\mathcal{P}_{\zeta}, r$ and $f_{\mathrm{NL}, \zeta}^{\text {equil. }}$ as a function of $\xi$, for various representative choices of the slow-roll parameter, $\epsilon$. However, since we are interested in particle production near the end of inflation we fix $\epsilon=1$. The total scalar power spectrum is plotted in figure 2 by using eq. (3.22). It is clear from the figure that when $\xi \sim 0.93$, the power spectrum can reach $4 \times 10^{-4}$ which is required value for long-lived PBHs formation.

From the left plot of figure 3 it is clear that the sourced GWs dominate over the vacuum ones for $\xi \gtrsim 2.41$ when $r=0.10$.

Using (3.25), we see that the $2 \sigma$ Planck 2015 limit (3.16) would enforce $\xi \lesssim 2.52$ (see right panel of figure 3).

As a result, our analysis show that the most stringent constraints on $\xi$ is derived from the non-production of DM PBHs at the end of inflation and the bounds from the bispectrum and the tensor-to-scalar ratio are weaker. However, the bound from the tensor-to-scalar 

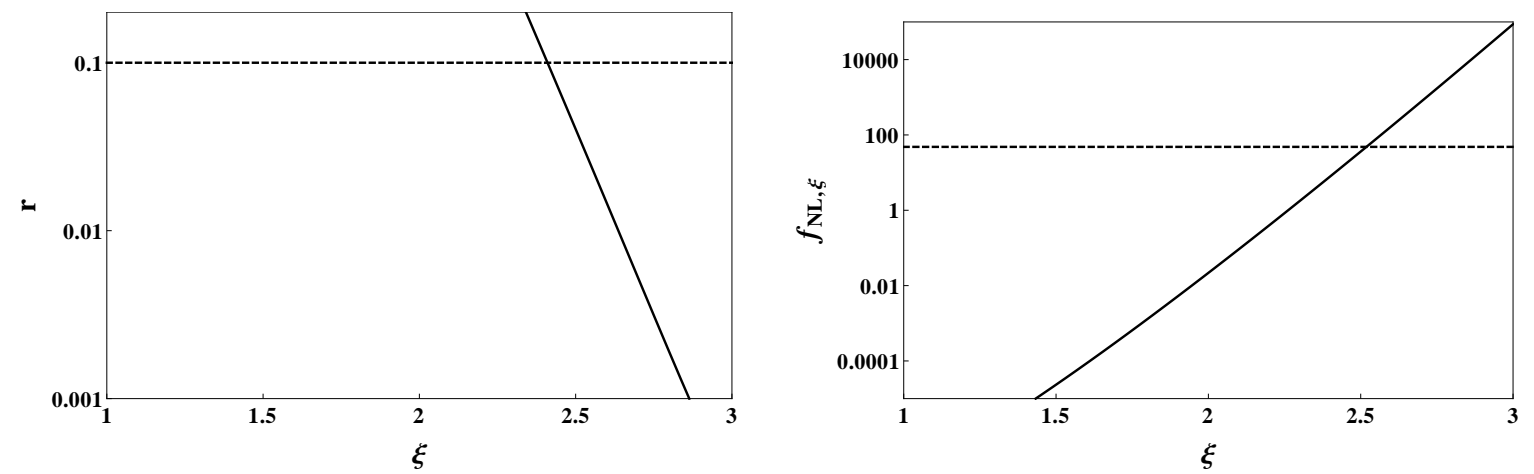

Figure 3. Predicted values for the tensor-to-scalar ratio, $r$ (left panel) and the equilateral $f_{\mathrm{NL}, \zeta}^{\text {equil. }}$ parameter (right plot) as a function of $\xi$ near the end of inflation. In left panel, the dashed line is Planck 2015 [38] upper bound $r<0.10$. The dashed line in the right plot is the $68 \%$ CL upper bound for $f_{\mathrm{NL}, \zeta}^{\text {equil. }}[39]$.

ratio is slightly more stringent than the NG bound. It is worth mentioning that we can also consider the PBHs formation during inflation (i.e. $\epsilon<1$ ), however the upper bounds on $\xi$ will be weaker.

\subsubsection{Model II: inflaton coupled gravitationally to the gauge field}

Above we discussed the possibility of DM PBHs where the gauge quanta are produced by a direct coupling between the inflaton and the gauge field. In this section we study the gauge production in a "hidden sector" where the vector and the pseudoscalar are only gravitationally coupled to the inflaton. In this case a rolling pseudoscalar sources gauge filed fluctuations continuously.

In the following we consider the system introduced in [14], in which a pseudoscalar field, $\chi$ couples to a $U(1)$ gauge filed

$$
\mathcal{L}=-\underbrace{\frac{1}{2} \partial_{\mu} \phi \partial^{\mu} \phi-V(\phi)}_{\text {inflaton sector }}-\underbrace{\frac{1}{2} \partial_{\mu} \chi \partial^{\mu} \chi-U(\chi)-\frac{1}{4} F_{\mu \nu} F^{\mu \nu}-\frac{\alpha}{4 f} \chi F_{\mu \nu} \tilde{F}^{\mu \nu}}_{\text {hidden sector }} .
$$

Here the assumption is that the $\chi-A_{\mu}$ sector has a negligible energy density with respect to the inflationary sector, and the inflaton sector and the hidden sector are coupled only gravitationally. In this case, gravitational coupling will transmit the effects of particle production in the hidden sector to the inflaton sector, and as in section 3.1.1, the production of gauge field fluctuations can provide a new source of scalar perturbations, complementary to the usual quantum vacuum fluctuations of the scalar part of the metric. The total spectrum of the scalar perturbations was found in [14] to be

$$
\mathcal{P}_{\zeta} \approx \mathcal{P}_{\zeta, \text { vac. }}\left(1+2.5 \times 10^{-6} \epsilon^{2} \mathcal{P}_{\zeta, \text { vac. }} X^{2}\right),
$$

therefore $\gamma_{\mathrm{s}} \simeq 2.5 \times 10^{-6}$. Note that here, $\xi \equiv \frac{\alpha}{2 f H} \dot{\chi}$.

Even if the vectors are not directly coupled to the inflaton, they generate tensor perturbations [14] which are chiral

$$
\begin{aligned}
& \mathcal{P}_{\mathrm{t}, \text { src. }}^{+}=2.13 \times 10^{-6} \mathcal{P}_{\mathrm{t}, \text { vac. }}^{2} X^{2}=5.41 \times 10^{-4} \epsilon^{2} \mathcal{P}_{\zeta, \text { vac. }}^{2} X^{2}, \\
& \mathcal{P}_{\mathrm{t}, \text { src. }}^{-}=4.44 \times 10^{-7} \mathcal{P}_{\mathrm{t} \text {, vac. }}^{2} X^{2}=1.14 \times 10^{-6} \epsilon^{2} \mathcal{P}_{\zeta, \text { vac. }}^{2} X^{2} .
\end{aligned}
$$




\begin{tabular}{|c|c|}
\cline { 2 - 2 } \multicolumn{1}{c|}{} & $\xi$ \\
\hline $\mathcal{P}_{\zeta}$ & 0.93 \\
\hline$r$ & 2.41 \\
\hline$f_{\mathrm{NL}, \zeta}^{\text {equil. }}$ & 2.86 \\
\hline$f_{\mathrm{NL}, \mathrm{t}}^{\text {equil. }}$ & 2.31 \\
\hline
\end{tabular}

Table 1. The obtained upper bounds on parameter $\xi$ by different observations. (See the main text for details.)

From eqs. (3.27) and (3.28) we can write the tensor-to-scalar ratio as

$$
r=16 \epsilon \frac{1+1.3 \times 10^{-6} \mathcal{P}_{\mathrm{t}, \text { vac. }} X^{2}}{1+2.5 \times 10^{-6} \epsilon^{2} \mathcal{P}_{\zeta, \text { vac. }} X^{2}} .
$$

The scalar bispectrum is given by [14]

$$
f_{\mathrm{NL}, \zeta}^{\text {equil. }} \approx 2.31 \times 10^{8} \epsilon^{3} \mathcal{P}_{\zeta, \text { vac. }}^{3} X^{3} .
$$

On the other hand, in this model, the tensor bispectrum also generates via the gravitational interaction with the gauge field, and its amplitude is estimated as [40]

$$
f_{\mathrm{NL}, \mathrm{t}}^{\text {equil. }} \approx 1.1 \times 10^{12} \epsilon^{3} \mathcal{P}_{\zeta, \text { vac. }}^{3} X^{3} .
$$

It is clear that the non-Gaussianity in the tensor perturbations is higher than the scalar perturbations.

In table 1, we report the obtained upper bounds on the particle production parameter, $\xi$ from the non-production of DM PBHs at the end of inflation and the observational bounds on the tensor-to-scalar ratio and the scalar and the tensor non-Gaussianity.

In this model also the bound on particle production parameter from the non-production of DM PBH is stronger than the ones derived from the (scalar and tensor) bispectrum and the tensor-to-scalar ratio. Note that the bound on $\xi$ from the tensor non-Gaussianity is stringent than the tensor-to-scalar ratio in this model.

\subsection{Scalar production}

In this final section we consider a very simple model where a slow-rolling inflaton, $\phi$ interacts with a massless scalar field, $\chi$ via the coupling [9]

$$
\mathcal{L}_{\text {int }}(\phi, \chi)=-\frac{g^{2}}{2}\left(\phi-\phi_{0}\right)^{2} \chi^{2},
$$

where $g$ is a coupling constant and $\phi_{0}$ is a constant field value. As $\phi(t)$ rolls down its potential, at the moment when $\phi=\phi_{0}$, the effective mass of $\chi$ - i.e. $m_{\chi}(t) \equiv g\left(\phi(t)-\phi_{0}\right)$ - becomes zero and its quanta can be copiously produced. This burst of particle production extracts energy from the condensate $\phi(t)$ and temporarily slows down the motion of the inflaton field. ${ }^{17}$ It was shown [6], the resonant extraction of even a small fraction of the energy of the

\footnotetext{
${ }^{17}$ It is also possible that several instances of particle production happens which gives rise to a new inflationary mechanism, called trapped inflation [41]. In this case multiple bursts of particle production leads many localized features in the power spectrum. And since the production of particles occurs several times per $e$ folding of inflation, the inflaton slows down, therefore inflation can occur even on a (relatively) steep potential. For more details see $[19,42]$.
} 


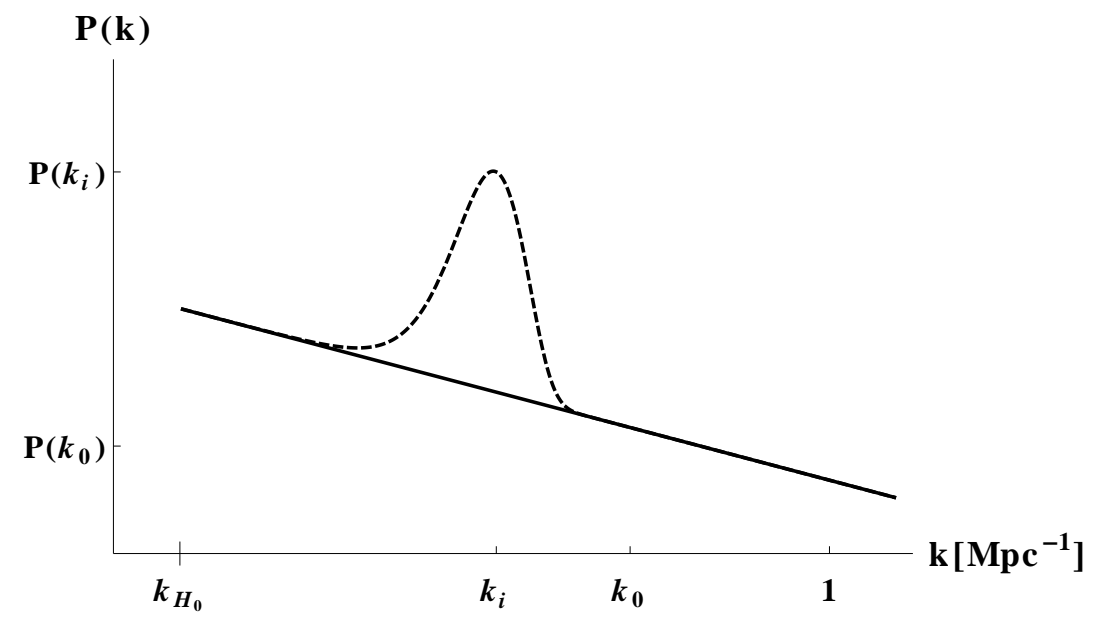

Figure 4. Power spectrum of curvature perturbations in the absence (solid curve) and the presence (dashed curve) of resonant particle creation in the Planck observational range; i.e. $k_{H_{0}} \lesssim k \lesssim 1$ $\mathrm{Mpc}^{-1}$. Resonant particle production produces a peak at $k_{i}=0.01 \mathrm{Mpc}^{-1}$.

inflaton field can alter the classical motion of the inflaton and generate a bump-like feature in the primordial power spectrum which in the model (3.32) can be fit with a following function

$$
\mathcal{P}_{\zeta, \text { src. }}(k) \sim k^{3} e^{-\frac{\pi}{2}\left(\frac{k}{k_{*}}\right)^{2}} .
$$

The location of the peak in wavenumber, $k_{*}$ depends on where the mass of the $\chi$ field passes through zero. ${ }^{18}$

It is straight forward to generalize this model to allow for multiple bursts of particle production during inflation [6]

$$
\mathcal{L}_{\text {int }}\left(\phi_{i}, \chi_{i}\right)=-\sum_{i=0}^{n} \frac{g_{i}^{2}}{2}\left(\phi-\phi_{i}\right)^{2} \chi_{i}^{2}
$$

In this case the contributed power spectrum from the burst of the produced $\chi_{i}$ particles is given by $[6]$

$$
\mathcal{P}_{\zeta, \text { src. }}(k)=\sum_{i=1}^{n} A_{i}\left(\frac{\pi e}{3}\right)^{3 / 2}\left(\frac{k}{k_{i}}\right)^{3} e^{-\frac{\pi}{2}\left(\frac{k}{k_{i}}\right)^{2}},
$$

where the constants, $A_{i}$ are the amplitudes of the power in the $i$-th bump which depend on the couplings, $g_{i}^{2}$ and, $k_{i}$ defines the location of features.

In this model, the sample bump in the power spectrum is illustrated in figure 4 and we take the bump to be located at $k_{i}=0.01 \mathrm{Mpc}^{-1}$.

As mentioned in section 2, if the power spectrum is $\mathcal{P}_{\zeta} \sim \mathcal{O}\left(10^{-4}\right)$, DM PBHs will be copiously produced in a scale corresponding to their masses. Here, to avoid their production in the CMB observational range (i.e. $k_{H_{0}} \lesssim k \lesssim 1 \mathrm{Mpc}^{-1}$ ), we can put an upper bound on the amplitude of the generated feature (and hence the couplings, $g_{i}$ ) due to the particle production; i.e. $A_{i} \lesssim 4 \times 10^{-4}$. Note that this bound is weaker than the one in [9], however,

\footnotetext{
${ }^{18}$ We should mention that the bump-like feature in the power spectrum can also produce in hybrid inflation models due to waterfall field transition. Therefore, PBHs can form in this case, too. For recent work in this subject, see [43].
} 
our result is completely model independent since we do not assume any specific potential for the inflaton field (i.e. the energy scale of inflation), therefore we can not put any bound on the abundance of produced (mono-chromatic) PBHs.

\section{Summary and conclusions}

In this work we reviewed the new class of inflation models where the inflaton field couples to an other field. The inclusion of this coupling is that it can produce the quanta of the coupled field. In this article we have studied two realizations of this scenario: where the coupled field is a vector or a scalar field. In the first case we studied the direct and gravitational coupling of the inflaton to the $U(1)$ gauge field. In this model, inflation is accompanied by the gauge quanta production and a strong rise of the curvature and tensor power spectrum amplitude at small scales along with equilateral non-Gaussianity is predicted. However, we have estimated that towards the end of inflation, i.e. on scales that are too small to be observed in the CMB, the power spectrum grows so much that the model may be ruled out because of the overproduction of (dark matter) primordial black holes. Our consideration was different from the studies in recent works [27-29] in two respects. Firstly, we did not assume any specific potential for the inflaton (or coupled) field. Secondly, we were only interested in PBHs with mass larger than $10^{15} \mathrm{~g}$ as candidate for DM. Since in the mentioned inflation models, the perturbations are highly non-Gaussian, we study the DM PBHs formation when the PDF of perturbations is non-Gaussian. Our analysis in section 2 showed that the power spectrum at the scale of DM PBHs formation should be $\mathcal{P}_{\zeta}\left(k_{\mathrm{PBH}}\right) \simeq 4 \times 10^{-4}$, which is two orders of magnitude smaller than the required scalar power spectrum when the perturbations are Gaussian. This means that the production probability of the non-evaporating PBHs when the perturbations are non-Gaussian is higher than the Gaussian case.

The main results when the inflaton coupled directly to the gauge field were shown in figures 2 and 3. From figure 2 it was clear that due to tachyonic instability of gauge field, an amplitude of the scalar power spectrum, $\mathcal{P}_{\zeta}$ can reach $\sim 10^{-4}$, which is required value for DM PBHs formation. Hence, we could put an upper bound on the particle production parameter; i.e. $\xi \lesssim 0.93$. The plots in figure 3 illustrated the fact that the upper bounds on the particle production parameter from the tensor perturbations and the bispectrum are weaker in comparison to the generated scalar perturbations.

In this paper we also discussed the gauge quanta production during inflation where quanta are produced by a gravitational coupling between the inflaton and the gauge field. In this case also the leading constraint on the particle production parameter comes from the non-production of long-lived PBHs. In this model, the bound on $\xi$ from the tensor bispectrum was stringent than the tensor-to-scalar ratio (see table 1).

In the second scenario where the inflaton field coupled to a scalar field, the model was free of DM PBHs overproduction in the CMB observational range if the amplitude of the generated bump in the scalar power spectrum, $A_{i}$ is less than $4 \times 10^{-4}$. This bound is weaker than the one in [9], however, our result was completely model independent.

As we mentioned, in this paper we did not consider any specific potential for the inflaton and the coupled field. It would be interesting to consider different inflationary potentials and study the particle production in these models. In this case one can put bound on the parameter(s) of the model such as the coupling constant via the non-production of PBHs. We leave this subject for a future work. It is also worth mentioning that in all studied models the 
produced PBHs can not give the right relic abundance of DM according to the observational results.

\section{Acknowledgments}

EE is grateful to the hospitality of the ICTP-SAIFR, São Paulo during her visit under the TWAS 2015 Fellowship for Research and Advanced Training. She is indebted to Eiichiro Komatsu for motivating the importance of this work during the MIAPP workshop "Cosmology after Planck". EE would like to thank Hassan Firouzjahi, Rafael Porto and Ivonne Zavala for their useful comments on the draft.

\section{References}

[1] D.H. Lyth and A. Riotto, Particle physics models of inflation and the cosmological density perturbation, Phys. Rept. 314 (1999) 1 [hep-ph/9807278] [INSPIRE].

[2] L. Kofman, A.D. Linde and A.A. Starobinsky, Reheating after inflation, Phys. Rev. Lett. 73 (1994) 3195 [hep-th/9405187] [INSPIRE].

[3] L. Kofman, A.D. Linde and A.A. Starobinsky, Towards the theory of reheating after inflation, Phys. Rev. D 56 (1997) 3258 [hep-ph/9704452] [INSPIRE].

[4] D.J.H. Chung, E.W. Kolb, A. Riotto and I.I. Tkachev, Probing Planckian physics: Resonant production of particles during inflation and features in the primordial power spectrum, Phys. Rev. D 62 (2000) 043508 [hep-ph/9910437] [INSPIRE].

[5] A.E. Romano and M. Sasaki, Effects of particle production during inflation, Phys. Rev. D 78 (2008) 103522 [arXiv:0809.5142] [INSPIRE].

[6] N. Barnaby, Z. Huang, L. Kofman and D. Pogosyan, Cosmological Fluctuations from Infra-Red Cascading During Inflation, Phys. Rev. D 80 (2009) 043501 [arXiv:0902.0615] [INSPIRE].

[7] E. Bugaev and P. Klimai, Trapping effects in inflation: blue spectrum at small scales, arXiv: 1511.06175 [INSPIRE].

[8] M.M. Anber and L. Sorbo, Naturally inflating on steep potentials through electromagnetic dissipation, Phys. Rev. D 81 (2010) 043534 [arXiv:0908.4089] [INSPIRE].

[9] N. Barnaby and Z. Huang, Particle Production During Inflation: Observational Constraints and Signatures, Phys. Rev. D 80 (2009) 126018 [arXiv:0909.0751] [InSPIRE].

[10] E. Pajer and M. Peloso, A review of Axion Inflation in the era of Planck, Class. Quant. Grav. 30 (2013) 214002 [arXiv: 1305.3557] [INSPIRE].

[11] R.Z. Ferreira and M.S. Sloth, Universal Constraints on Axions from Inflation, JHEP 12 (2014) 139 [arXiv: 1409.5799] [INSPIRE].

[12] W.D. Garretson, G.B. Field and S.M. Carroll, Primordial magnetic fields from pseudoGoldstone bosons, Phys. Rev. D 46 (1992) 5346 [hep-ph/9209238] [INSPIRE].

[13] N. Barnaby and M. Peloso, Large NonGaussianity in Axion Inflation, Phys. Rev. Lett. 106 (2011) 181301 [arXiv: 1011.1500] [INSPIRE].

[14] N. Barnaby, J. Moxon, R. Namba, M. Peloso, G. Shiu and P. Zhou, Gravity waves and non-Gaussian features from particle production in a sector gravitationally coupled to the inflaton, Phys. Rev. D 86 (2012) 103508 [arXiv: 1206.6117] [InSPIRE].

[15] N. Barnaby, R. Namba and M. Peloso, Phenomenology of a Pseudo-Scalar Inflaton: Naturally Large NonGaussianity, JCAP 04 (2011) 009 [arXiv:1102.4333] [INSPIRE]. 
[16] L. Sorbo, Parity violation in the Cosmic Microwave Background from a pseudoscalar inflaton, JCAP 06 (2011) 003 [arXiv:1101.1525] [InSPIRE].

[17] M. Drees and E. Erfani, Running-Mass Inflation Model and Primordial Black Holes, JCAP 04 (2011) 005 [arXiv: 1102.2340] [INSPIRE].

[18] M. Drees and E. Erfani, Running Spectral Index and Formation of Primordial Black Hole in Single Field Inflation Models, JCAP 01 (2012) 035 [arXiv:1110.6052] [INSPIRE].

[19] J.L. Cook and L. Sorbo, Particle production during inflation and gravitational waves detectable by ground-based interferometers, Phys. Rev. D 85 (2012) 023534 [Erratum ibid. D 86 (2012) 069901] [arXiv:1109.0022] [INSPIRE].

[20] K. Freese, J.A. Frieman and A.V. Olinto, Natural inflation with pseudo-Nambu-Goldstone bosons, Phys. Rev. Lett. 65 (1990) 3233 [InSPIRE].

[21] N. Barnaby, E. Pajer and M. Peloso, Gauge Field Production in Axion Inflation: Consequences for Monodromy, non-Gaussianity in the CMB and Gravitational Waves at Interferometers, Phys. Rev. D 85 (2012) 023525 [arXiv:1110.3327] [INSPIRE].

[22] LiGO Scientific collaboration, J. Aasi et al., Advanced LIGO, Class. Quant. Grav. 32 (2015) 074001 [arXiv: 1411.4547] [INSPIRE].

[23] VIRGO collaboration, F. Acernese et al., Advanced Virgo: a second-generation interferometric gravitational wave detector, Class. Quant. Grav. 32 (2015) 024001 [arXiv:1408.3978] [INSPIRE].

[24] S. Mukohyama, R. Namba, M. Peloso and G. Shiu, Blue Tensor Spectrum from Particle Production during Inflation, JCAP 08 (2014) 036 [arXiv:1405.0346] [INSPIRE].

[25] W.H. Press and P. Schechter, Formation of galaxies and clusters of galaxies by selfsimilar gravitational condensation, Astrophys. J. 187 (1974) 425 [INSPIRE].

[26] T. Harada, C.-M. Yoo and K. Kohri, Threshold of primordial black hole formation, Phys. Rev. D 88 (2013) 084051 [arXiv: 1309.4201] [INSPIRE].

[27] C.-M. Lin and K.-W. Ng, Primordial Black Holes from Passive Density Fluctuations, Phys. Lett. B 718 (2013) 1181 [arXiv:1206.1685] [INSPIRE].

[28] A.D. Linde, S. Mooij and E. Pajer, Gauge field production in supergravity inflation: Local non-Gaussianity and primordial black holes, Phys. Rev. D 87 (2013) 103506 [arXiv: 1212.1693] [INSPIRE].

[29] E. Bugaev and P. Klimai, Axion inflation with gauge field production and primordial black holes, Phys. Rev. D 90 (2014) 103501 [arXiv:1312.7435] [INSPIRE].

[30] B.J. Carr and S.W. Hawking, Black holes in the early Universe, Mon. Not. Roy. Astron. Soc. 168 (1974) 399 [INSPIRE].

[31] Y.B. Zel'dovich and I.D. Novikov, The Hypothesis of Cores Retarded during Expansion and the Hot Cosmological Model, Sov. Astron. 10 (1967) 602 [Astron. Zh. 43 (1966) 758].

[32] B.J. Carr, K. Kohri, Y. Sendouda and J. Yokoyama, New cosmological constraints on primordial black holes, Phys. Rev. D 81 (2010) 104019 [arXiv:0912.5297] [InSPIRE].

[33] T. Nakama and T. Suyama, Primordial black holes as a novel probe of primordial gravitational waves, Phys. Rev. D 92 (2015) 121304(R) [arXiv: 1506.05228] [INSPIRE].

[34] S. Hawking, Gravitationally collapsed objects of very low mass, Mon. Not. Roy. Astron. Soc. 152 (1971) 75 [INSPIRE].

[35] B.J. Carr, The Primordial black hole mass spectrum, Astrophys. J. 201 (1975) 1 [INSPIRE].

[36] Planck collaboration, P.A.R. Ade et al., Planck 2015 results. XIII. Cosmological parameters, arXiv: 1502.01589 [INSPIRE]. 
[37] D.H. Lyth, The hybrid inflation waterfall and the primordial curvature perturbation, JCAP 05 (2012) 022 [arXiv:1201.4312] [INSPIRE].

[38] Planck collaboration, P.A.R. Ade et al., Planck 2015 results. XX. Constraints on inflation, arXiv: 1502.02114 [INSPIRE].

[39] PLAnCK collaboration, P.A.R. Ade et al., Planck 2015 results. XVII. Constraints on primordial non-Gaussianity, arXiv: 1502.01592 [INSPIRE].

[40] J.L. Cook and L. Sorbo, An inflationary model with small scalar and large tensor nonGaussianities, JCAP 11 (2013) 047 [arXiv:1307.7077] [INSPIRE].

[41] D. Green, B. Horn, L. Senatore and E. Silverstein, Trapped Inflation, Phys. Rev. D 80 (2009) 063533 [arXiv:0902.1006] [INSPIRE].

[42] W. Lee, K.-W. Ng, I.-C. Wang and C.-H. Wu, Trapping effects on inflation, Phys. Rev. D 84 (2011) 063527 [arXiv:1101.4493] [INSPIRE].

[43] S. Clesse and J. García-Bellido, Massive Primordial Black Holes from Hybrid Inflation as Dark Matter and the seeds of Galaxies, Phys. Rev. D 92 (2015) 023524 [arXiv:1501.07565] [INSPIRE]. 